\title{
Full-Carpet Design of a Low-Boom Demonstrator Concept
}

\author{
Irian Ordaz* \\ NASA Langley Research Center, Hampton, VA 23681 \\ Mathias Wintzer ${ }^{\dagger}$ \\ Analytical Mechanics Associates, Inc., Hampton, VA 23666 \\ Sriram K. Rallabhandi ${ }^{\ddagger}$ \\ National Institute of Aerospace, Hampton, VA 23666
}

\begin{abstract}
The Cart3D adjoint-based design framework is used to mitigate the undesirable off-track sonic boom properties of a demonstrator concept designed for low-boom directly under the flight path. First, the requirements of a Cart3D design mesh are determined using a highfidelity mesh adapted to minimize the discretization error of the CFD analysis. Low-boom equivalent area targets are then generated at the under-track and one off-track azimuthal position for the baseline configuration. The under-track target is generated using a trimfeasible low-boom target generation process, ensuring that the final design is not only low-boom, but also trimmed at the specified flight condition. The off-track equivalent area target is generated by minimizing the A-weighted loudness using an efficient adjointbased approach. The configuration outer mold line is then parameterized and optimized to match the off-body pressure distributions prescribed by the low-boom targets. The numerical optimizer uses design gradients which are calculated using the Cart3D adjointbased design capability. Optimization constraints are placed on the geometry to satisfy structural feasibility. The low-boom properties of the final design are verified using the adaptive meshing approach. This analysis quantifies the error associated with the CFD mesh that is used for design. Finally, an alternate mesh construction and target positioning approach offering greater computational efficiency is demonstrated and verified.
\end{abstract}

\section{Introduction}

Commercial interest in supersonic travel dates to as early as the 1950s. Since then, several efforts such

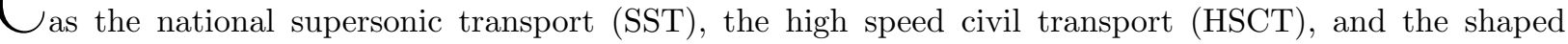
sonic boom demonstrator (SSBD) have improved our understanding of commercial supersonic flight and the sonic boom phenomena associated with it. Two aspects which affect the commercial viability of supersonic flight include increased operating cost, and the overland supersonic flight ban, which severely restricts airline operating markets. Recent efforts by NASA, industry, and academia have focused on developing new methods and tools to better address the challenge of reducing the sonic boom generated by a supersonic aircraft. The research conducted to better understand and mitigate the sonic boom phenomena aims to increase the general public acceptance of supersonic flight overland. NASA continues to work closely with national and international bodies (such as the FAA and ICAO) to establish requirements, which may help reconsideration of the overland supersonic flight ban. ${ }^{1}$

The work presented in this paper is part of an effort to demonstrate that sonic booms can be reduced to publicly acceptable levels, while leveraging recent and more practical computational techniques for aerodynamic analysis and design. Application of adjoint methods for efficient calculation of design sensitivities has become more practical for conceptual and preliminary design. Design frameworks based on adjoint methods

*Aerospace Engineer, Aeronautics Systems Analysis Branch, Member AIAA.

${ }^{\dagger}$ Engineer, Aeronautics Systems Analysis Branch, Senior Member AIAA.

¥Associate Research Fellow, Aeronautics Systems Analysis Branch, Associate Fellow AIAA. 
allow design with a larger number of design variables without a significant increase in computational cost. Adjoint methods also provide a better understanding of the design space for more complex problems. This approach is applied to the full-carpet design of a NASA-developed low-boom flight demonstrator concept. ${ }^{2}$

The paper contains six sections, including the introduction section. Section II discusses the analysis, design, and optimization tools that are used during the design process. Section III discusses the design formulation, including a description of the baseline configuration, the calibration of the design mesh, the generation of low-boom targets, and the geometry parameterization and numerical optimization approach. Section IV describes the parametric schemes, design procedures, and results of the optimization process. Section V provides high-fidelity verification results to assess the adequacy of the CFD mesh for the final design. Finally, a summary of the work, and future work is discussed in Section VI.

\section{Description of Analysis, Design, and Optimization Tools}

The CFD analysis is conducted with Cart3D,,$^{3,4}$ an inviscid CFD analysis package that is geared toward conceptual and preliminary aerodynamic design. The package allows for quick and automated Cartesianbased volume mesh generation, and CFD solutions for complex geometries. The volume mesh used for sonic boom analysis and design is rotated by the Mach angle to align the shocks with the computational grid along the $x$ - $z$ plane at $y=0$. Numerical dissipation and discretization error in the computational domain can be reduced using hand-tailored refinement boxes and adjoint-based mesh adaptation, respectively. This region typically extends 3-5 body lengths away from the aircraft, where three-dimensional effects are sufficiently resolved, and use of 2-D propagation algorithms are acceptable for sonic boom design.

The off-body pressure distribution $\left(\Delta p / p_{\infty}\right)$ calculated with Cart3D is propagated with the atmospheric propagation code $\mathrm{sBOOM}^{5}$ to obtain the ground signatures $(\Delta p)$, and perceived level of loudness (PLdB). By solving the augmented Burgers equation, sBOOM takes into account atmospheric losses due to nonlinearity, molecular relaxation, and thermo-viscous absorption. All propagation results presented in this paper assume an atmosphere with no winds or turbulence. Recent work by Rallabhandi ${ }^{6}$ extends the capabilities of sBOOM to allow the calculation of adjoint-based sonic boom sensitivities for equivalent area $\left(A_{\mathrm{e}}\right)$ distributions. This capability is used here to generate an off-track low-boom target $\Delta p / p_{\infty}$ in the vicinity of the baseline solution.

The Cart3D analysis and design framework ${ }^{7}$ also allows the calculation of design sensitivities ${ }^{7,8}$ for a large number of design variables using a single flow and adjoint solution. The parametric modeler JAGUAR is used to define the conceptual geometry, generate the surface mesh, and provide mesh sensitivity to the Cart3D design framework. The sensitivity of the surface mesh with respect to design variables is calculated with JAGUAR through finite differencing. A more detailed description of the geometry modeling approach for the configuration in this paper is provided in a companion paper. ${ }^{10}$ The design gradients calculated by the Cart3D framework are provided to the gradient-based Sparse Nonlinear OPTimizer (SNOPT). ${ }^{9,11}$ SNOPT uses a Sequential Quadratic Programming (SQP) algorithm to solve an inverse design problem, which is described in more detail in Section IIIC.

\section{Design Formulation}

\section{A. Description of Baseline Configuration and Parametric Model}

The baseline configuration, shown in Fig. 1, is similar to the baseline configuration in Ref. 10, which described the design of this demonstrator concept to achieve low-boom directly below the flight path. The configuration consists of fuselage, nacelle, and vertical and horizontal tail geometries identical to those of the baseline described in Ref. 10 to avoid unnecessary bias toward under-track shaping. However, it also retains the tail pod, and wing shape of the final shaped configuration from Ref. 10 because it has characteristics favorable for trim, and can already accommodate sufficient volume to store the main landing gear. The tail pod component is used strictly for sonic boom mitigation, and provides additional design authority at the end of the configuration.

All airfoils which define the wing and horizontal tail of the baseline configuration are modeled as Kulfan ${ }^{12}$ sections to provide more design authority with the wing. The concept is powered by a single General Electric F404 engine, which is modeled as a flow-through nacelle during low-boom design to reduce the complexity of the CFD analysis.

The mission design point is the start of cruise at an altitude of 51,700 ft, Mach 1.6, angle of attack of $3.28 \mathrm{deg}$, and weight of 21,000 lb. Figure 2 shows the off-track sonic boom analysis for our baseline, and 


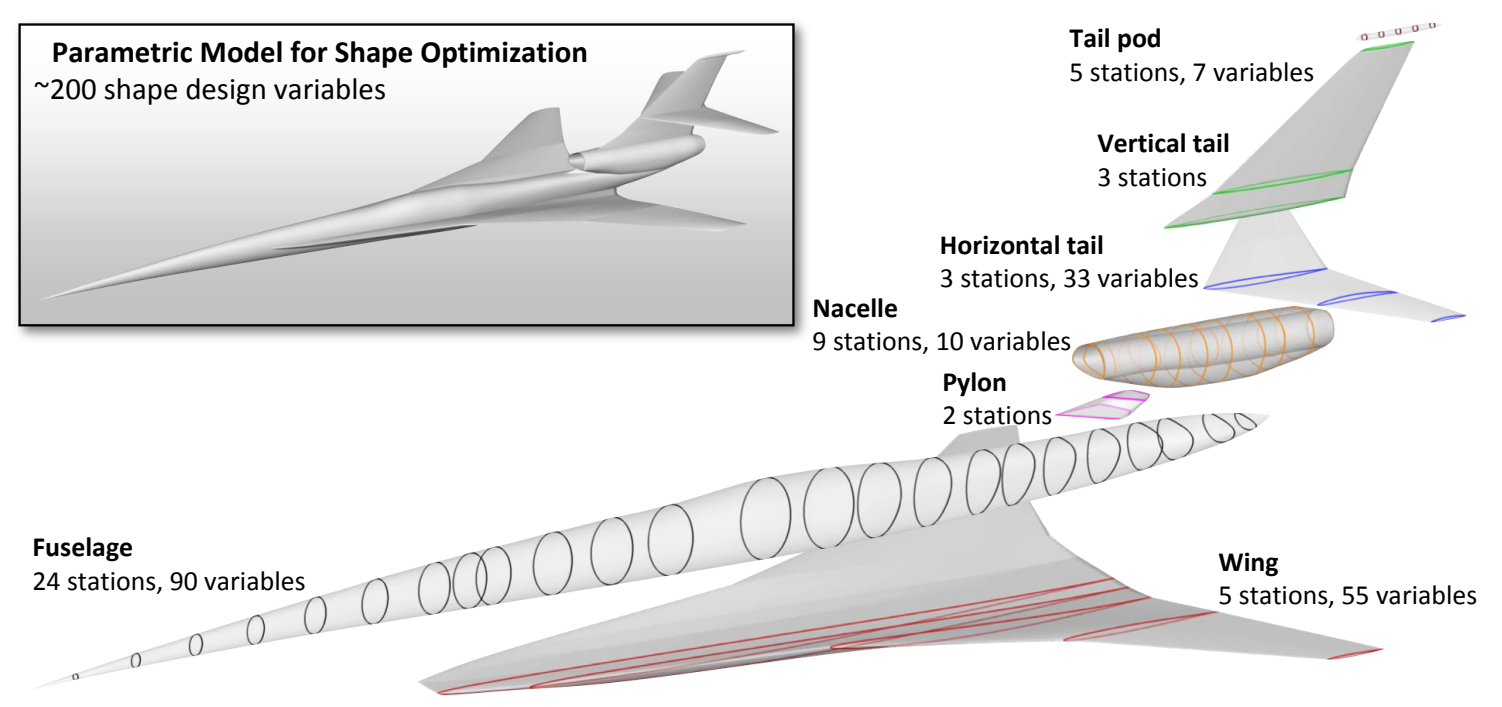

Figure 1. Jaguar geometry model and parametric sections.

the final design configuration that was shaped for low-boom directly below the flight path in Ref. 10. Here, the PLdB of the shaped configuration in the under-track $(0 \mathrm{deg})$ direction was reduced from 81.6 to 73.8 . Figure 2 shows that the loudness for this shaped configuration was also reduced between the under-track, and an off-track azimuth angle $(\phi)$ of $20 \mathrm{deg}$. This range represents a lateral distance of approximately 21,000 ft $(3.5 \mathrm{nmi})$. It is also possible to see that the loudness remains either unchanged or higher than that of the baseline configuration at off-track angles of $25 \mathrm{deg}$ and greater. Good off-track sonic boom performance between $25 \mathrm{deg}$ and $45 \mathrm{deg}$ is especially desirable because this represents a significantly larger lateral distance of approximately $41,000 \mathrm{ft}(6.7 \mathrm{nmi})$.

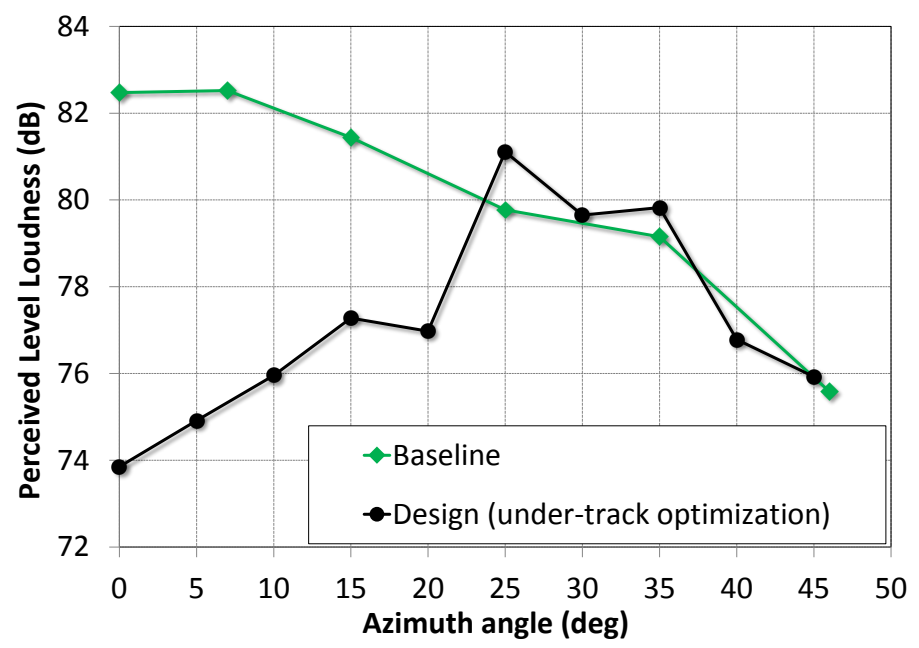

Figure 2. Off-track loudness distribution of the baseline configuration and its re-design for low-boom below the flight path.

\section{B. Calibration of CFD Mesh used for Design}

The CFD mesh used for design must allow accurate prediction of the sonic boom phenomena but also limit the computational resources required. The computational domain must also extend in the spanwise direction to accurately compute the aerodynamic characteristics in the off-track region. To achieve a balance between accuracy and computational cost, the design mesh is calibrated to ensure that the solution obtained with a prescribed mesh is consistent with an adapted mesh solution. Once the design mesh is calibrated, it remains 
fixed throughout the design process.

For the design mesh, a hand-tailored sonic boom refinement box is placed in the computational domain to capture the physics in the under-track, as well as the off-track region. The spanwise size of this highrefinement region is limited to a radial distance $(h / L)$ of two body lengths away from the aircraft along the $25 \mathrm{deg}$ azimuthal direction. This results in a 30 million cell CFD mesh, which captures the sonic boom characteristics at the boundary of the off-track region that is beyond the scope of the under-track optimization $^{10}$ (see Fig. 2). The error associated with propagating $\Delta p / p_{\infty}$ from $h / L=2$ is quantified through Cart3D analysis in Ref. 10, and the corresponding ground signatures show that the error is acceptable for the design of this configuration. A comparison of $\Delta p / p_{\infty}$ calculated with Cart3D for the baseline configuration at $h / L=2$ using a 44 million cell adapted mesh and the 30 million cell design mesh is given in Fig. 3. This plot shows good agreement at both under- and 25 deg off-track directions.

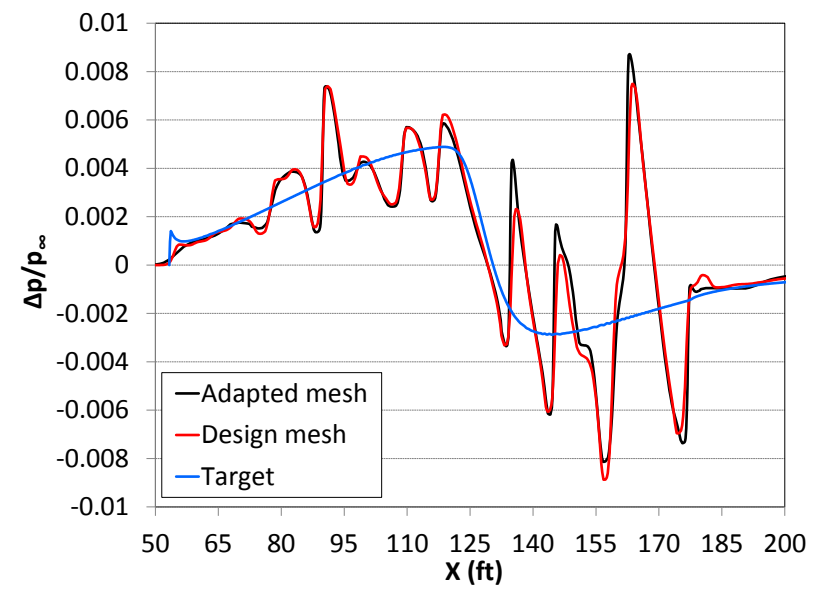

(a) Under-track.

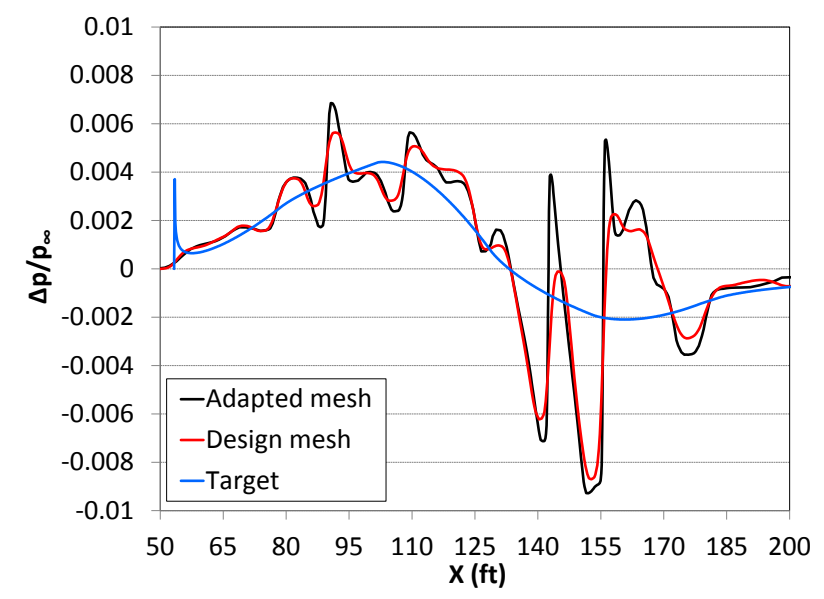

(b) 25 deg off-track.

Figure 3. Comparison of $\Delta p / p_{\infty}$ between the adapted and design meshes for the baseline configuration at $h / L=2$.

\section{Generation of Low-Boom Targets}

The inverse design approach, often used for sonic boom optimization takes advantage of the natural decoupling in the sonic boom analysis. ${ }^{8}$ Inverse design requires optimization of the outer mold line (OML) of a configuration to tailor the flowfield, and match a prescribed target $\Delta p / p_{\infty}$ that results in low-boom characteristics when the pressure signal reaches the ground. This approach can be extended to match the $\Delta p / p_{\infty}$ at under- and off-track directions using a combined, weighted metric. Low-boom $\Delta p / p_{\infty}$ targets need to be generated for these additional off-track directions at several body lengths away from the aircraft.

For this particular design, only two equally weighted azimuthal directions are chosen for inverse design: the under-track and $25 \mathrm{deg}$ azimuthal directions. The inverse design to match an under-track target is critical as the chosen trim-feasible target described in Ref. 2 implicitly drives the design to not only lowboom, but also to a trimmed state at the start of cruise. Limiting the multi-objective design to only two azimuthal directions reduces the complexity, and the risk of over-constraining the optimization problem. A potential risk of this simplification is that there is less control over the sonic boom characteristics at other azimuthal directions of the sonic boom carpet. Improvement of the sonic boom carpet in one region may result in degradation in a different region. This problem is configuration dependent, and targets should only be prescribed at additional off-track directions when necessary.

The $\Delta p / p_{\infty}$ calculated with the design mesh along $\phi=25 \operatorname{deg}$ at $h / L=2$ is used as the starting point for the generation of the off-track low-boom target. The pressure distribution is first converted to the $A_{\mathrm{e}}$ domain, and a cubic spline is fitted to the $A_{\mathrm{e}}$ distribution. The adjoint-based optimization process presented by Rallabhandi ${ }^{6}$ is then used to minimize the A-weighted loudness by varying the spline control points that define the $A_{\mathrm{e}}$. The A-weighted loudness has been found to correlate well with PLdB in the range of loudness levels pertinent to this paper. ${ }^{13}$ The under- and off-track target $\Delta p / p_{\infty}$ are shown in Figs. 3(a) and 3(b), and the corresponding PLdB values for their ground signatures are 66 and 72 , respectively. 


\section{Design Procedures and Results}

The shape design variables are decomposed into three groups (see Fig. 4), which simplify the design problem by reducing the number of design variables that are optimized at one time. The decomposition is possible because in the supersonic flight regime, the governing compressible flow equations reduce to hyperbolic form. As a result, the domain of influence and dependence of any design change is well understood. The practical design implication is that aerodynamic effects associated with design variable changes in a group only propagate downstream within the Mach cone of influence. Note that Fig. 4 is only a notional diagram, and in some cases there exists a certain degree of overlap between the variable groups. In an effort to be concise, only the optimization procedures that exhibit significant improvement are presented.

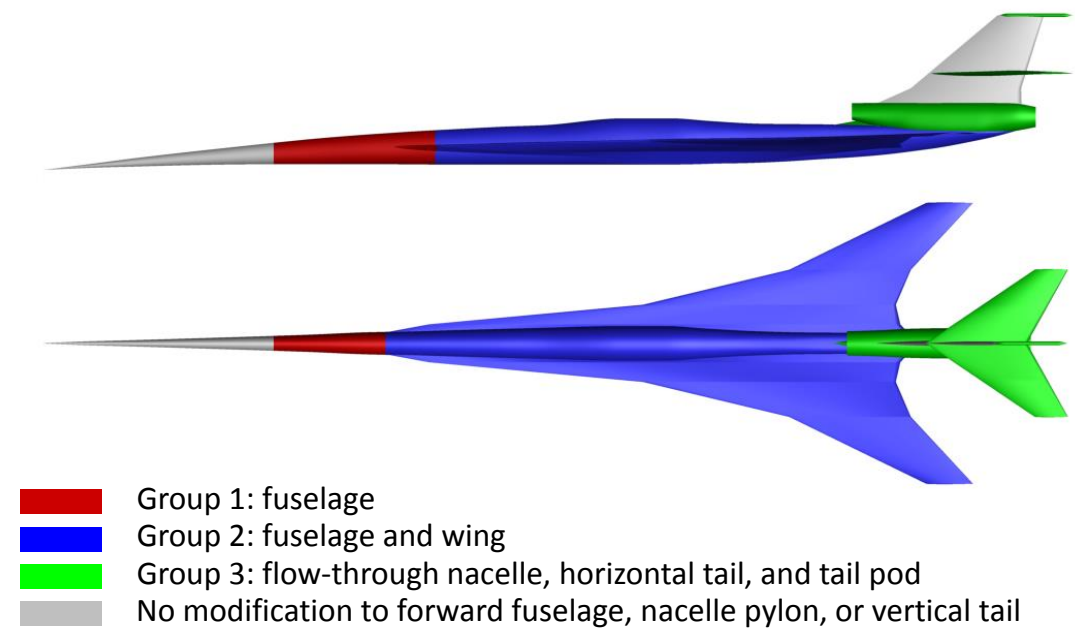

Figure 4. Grouping of design variables.

An initial contour map of the density adjoint calculated by Cart3D for the baseline configuration is provided in Fig. A-1 of the Appendix. This figure is useful because it identifies regions of the configuration, which can be used to shape and match the target $\Delta p / p_{\infty}$ at this point in the design. The objective of the first design procedure with group 1 design variables is to shape the forward portion of the fuselage ahead of the wing. This variable group consists of 20 design variables corresponding to circular and elliptical sections. In the case of elliptical sections, both width and height parameters are allowed to vary. Note that this variable group does not include the foremost portion of the fuselage or nose cone, which is dominated by the volume component effects of the classical $A_{\mathrm{e}}$. Rather, its shape is specified early on in the design process by matching the volume $A_{\mathrm{e}}$ to the target $A_{\mathrm{e}}$. The resulting $\Delta p / p_{\infty}$ for this design procedure exhibits a good match to the target, as shown in Fig. 5 for both the under- and off-track directions.

The fore and aft PLdB calculation is a way to decompose the effect of the fore and aft portions of the configuration on the overall PLdB. These values are calculated by splitting the ground signature into fore, and aft domains at the first location where the pressure changes sign. It is used only as a guide to better understand which region of the configuration dominates PLdB, and where further shaping is required. The fore PLdB is improved from 79 to 73.5 in the under-track direction, and 77.3 to 71 in the 25 deg off-track direction.

The next design procedure continues downstream to variable group 2, which consists of approximately 58 design variables. The wing is parameterized at 5 airfoil sections, with $10 \mathrm{Kulfan}$ parameters defining the upper and lower thickness distribution. Constraints are placed on the minimum thickness, area (between $20 \%$ and $60 \%$ chord), and leading and trailing edge angles of each airfoil section. ${ }^{10}$ The fuselage sections in group 2 are modeled as super-ellipses but only the width and lower heights are allowed to vary. A comparison of $\Delta p / p_{\infty}$ for this design procedure is provided in Fig. 6. Note that the distributions denoted as baseline in all figures depicting $\Delta p / p_{\infty}$ and $\Delta p$ correspond to that of the initial baseline, and not the best design of the previous design procedure. The aft PLdB improves from 80 to 72.5 in the under-track direction, and 78.5 to 72.4 in the off-track direction, while the fore PLdB remains relatively unchanged.

The resulting wing deformation is presented in Fig. 7 for three selected airfoil sections. This figure is scaled in the vertical direction as $z / x=2$ to provide a better visualization of the airfoil section deformation. 


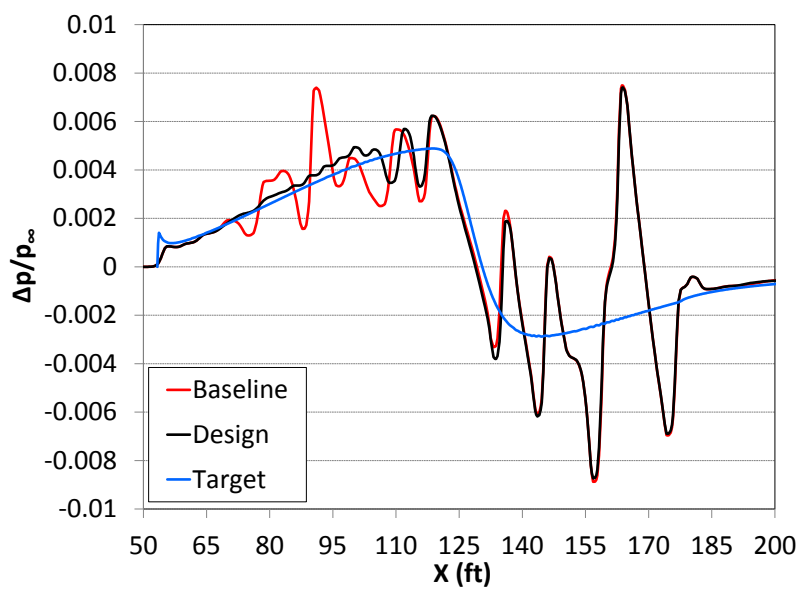

(a) Under-track.

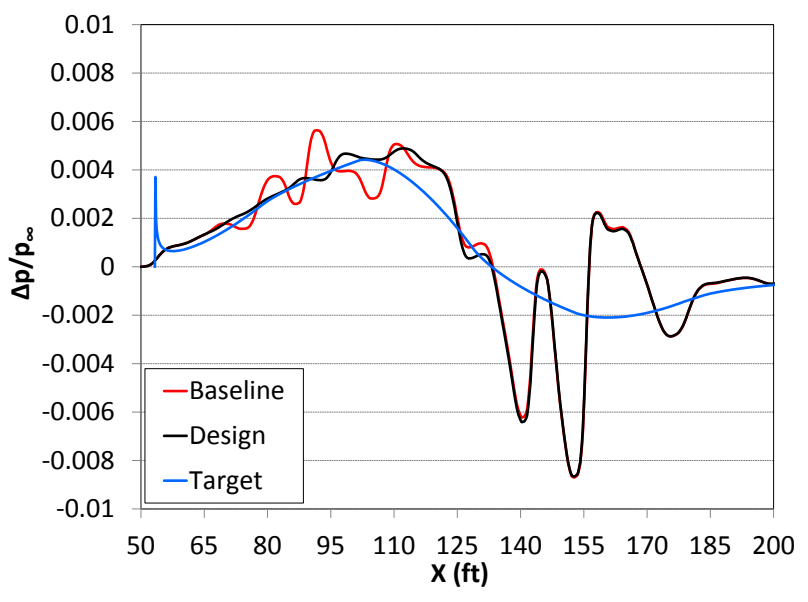

(b) 25 deg off-track.

Figure 5. Comparison of $\Delta p / p_{\infty}$ for shaping of forward fuselage at $h / L=2$.

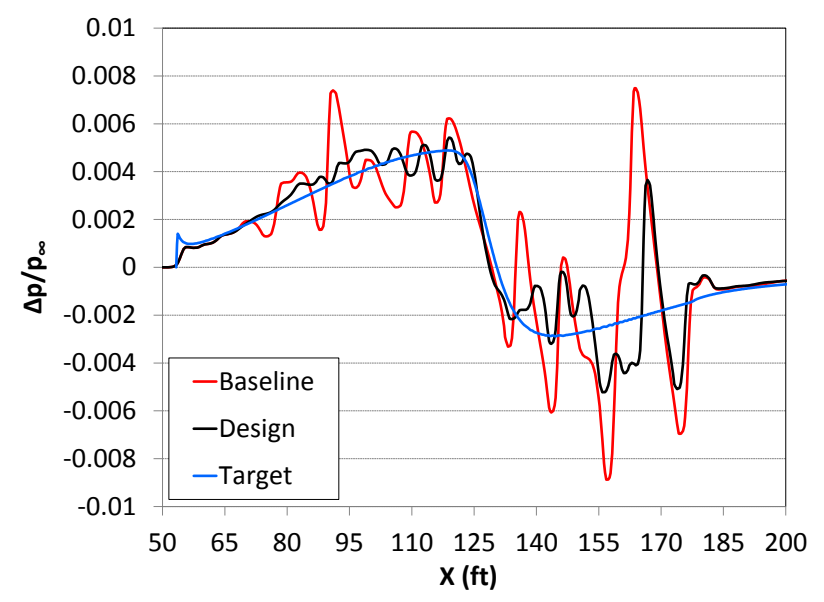

(a) Under-track.

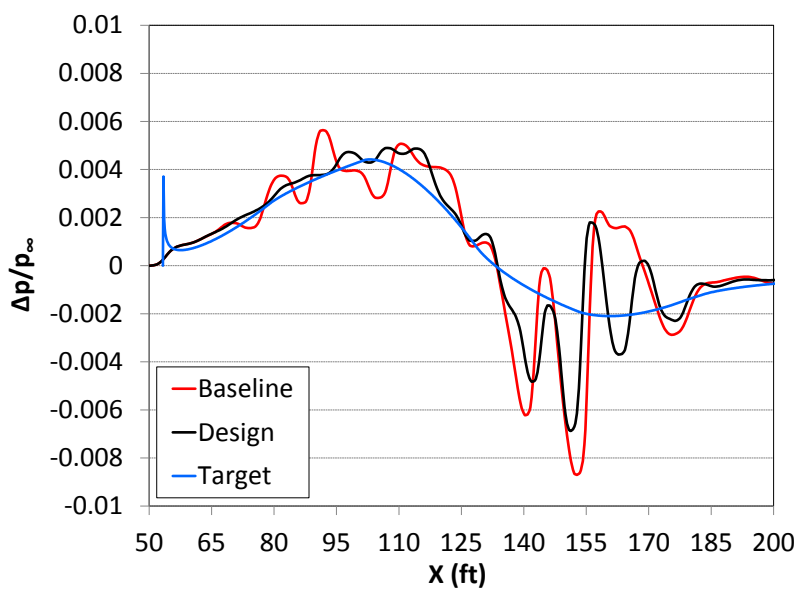

(b) 25 deg off-track.

Figure 6. Comparison of $\Delta p / p_{\infty}$ for shaping of wing and aft fuselage at $h / L=2$.

Several problems emerged during this design procedure. First, it was discovered that the optimizer was driving the thickness of the wing at the $22 \%$ chord location near the midspan section to zero. As a result, an additional minimum thickness constraint was added at this location to prevent this undesirable feature from progressing further. It was also found that the changes necessary to achieve low-boom in the under-track direction led to compromises in the sonic boom at the $25 \mathrm{deg}$ off-track direction. This is evident by the nature of the $\Delta p / p_{\infty}$ in the region of $x=140 \mathrm{ft}$ in Fig. 6. Here, the under-track $\Delta p / p_{\infty}$ has a system of shocks that oscillates close to the target $\Delta p / p_{\infty}$, however, the off-track $\Delta p / p_{\infty}$ exhibits strong expansions, which track well below the off-track $\Delta p / p_{\infty}$ target in the same region. It was discovered through experimentation with different sets of shaping variables that for this layout, the decoupling of the aerodynamics effects between the under- and 25 deg off-track directions was difficult, and always resulted in compromised solutions.

Attempts were also made to generate a low-boom target for the off-track direction that was more feasible (closer in the $A_{\mathrm{e}}$ domain) for this configuration. However, the loudness level for all candidate targets was greater than 75 PLdB. Further studies are needed to better understand how to decouple the aerodynamic effects due to shape deformation for the two azimuthal directions. It may also be possible to extend the trim-feasible target generation process that was presented in Ref. 2 for the under-track direction to account for the sonic boom at off-track directions.

Finally, the design variables within group 3 are activated. This group includes variables of the nacelle, 


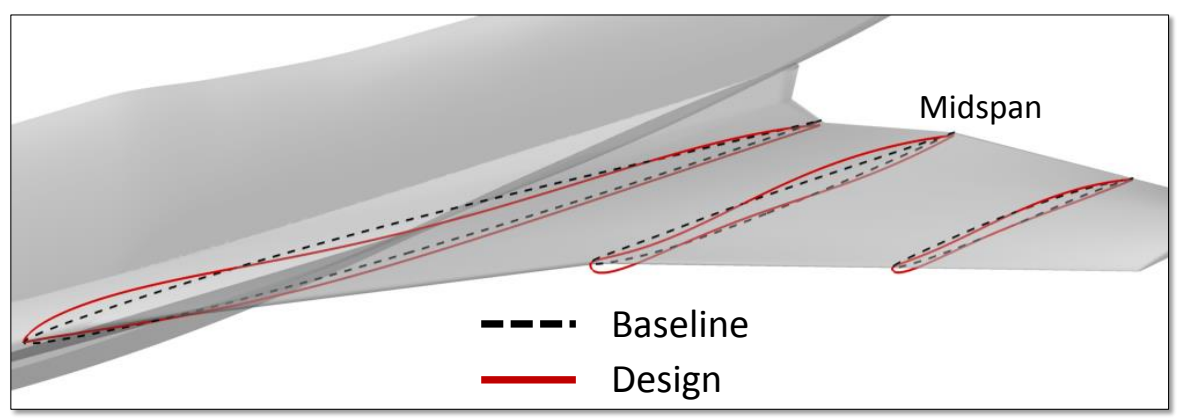

Figure 7. Comparison of wing airfoil sections for the baseline and final design configurations $(z / x=2$ scaling).

horizontal tail, tail pod, and the three aftmost sections of the fuselage. The nacelle and the three aftmost sections of the fuselage are modeled as super-ellipses. The first and last nozzle sections of the nacelle remain unchanged but the intermediate sections are allowed to vary in width. A minimum width constraint is imposed on the nacelle so that it is only allowed to increase in width relative to the baseline configuration. This constraint guarantees the volume necessary for the F404 engine and its accessories, and also prevents incursions of the nacelle OML into the inlet. The fuselage sections are allowed to vary in both width and lower height. The three sections which define the horizontal tail are modeled with Kulfan parametric sections. Minimum constraints on thickness, area, and leading and trailing edge angles are also placed on all airfoil sections of the horizontal tail.

A comparison of $\Delta p / p_{\infty}$ for the baseline and final design is shown in Fig. 8. These figures show that the optimization strategy is successful in shaping the aft region of the configuration. The large aft shock in the under-track direction has been successfully resolved. The aftmost shock located at $x=162 \mathrm{ft}$ in the $25 \mathrm{deg}$ direction has been eliminated, and while the rest of the shocks remain, their strengths have been reduced. The $\Delta p$ shown in Figs. 9(a) and 9(b) are calculated by propagating the $\Delta p / p_{\infty}$ for the under- and 25 deg off-track directions given in Figs. 8(a) and 8(b). The final PLdB values at the under- and off-track directions are 76.4 and 76.8 , respectively.

It is interesting to note that while the $\Delta p / p_{\infty}$ match in the under-track direction is better than that in the 25 deg off-track direction, the latter has a shock structure which behaves well during propagation. Although large in strength relative to the under-track shock structure; the two aft shocks in the off-track direction never coalesce, and in fact, have more time to dissipate as they propagate a larger distance through the atmosphere than those in the under-track direction. The large number of weak shocks in the under-track direction coalesce into a single shock which is stronger than the two aft shocks in the off-track direction.

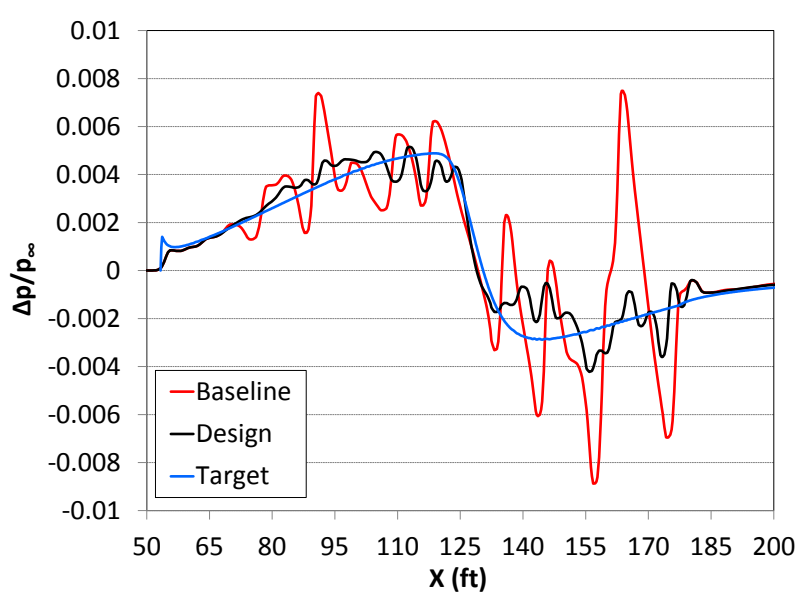

(a) Under-track.

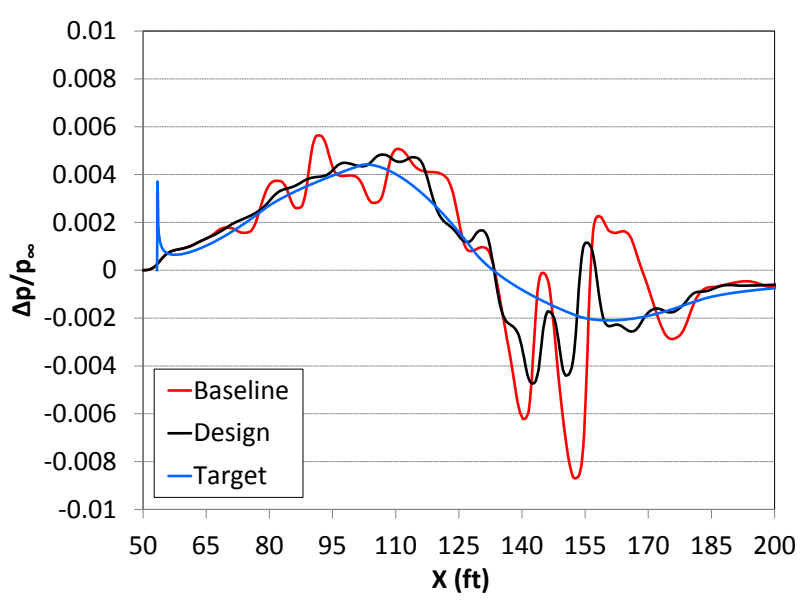

(b) 25 deg off-track.

Figure 8. Comparison of $\Delta p / p_{\infty}$ for shaping of nacelle, horizontal tail, and tail pod at $h / L=2$.

The more significant shape deformations for this design procedure are provided in Fig. 10 for the fuselage 


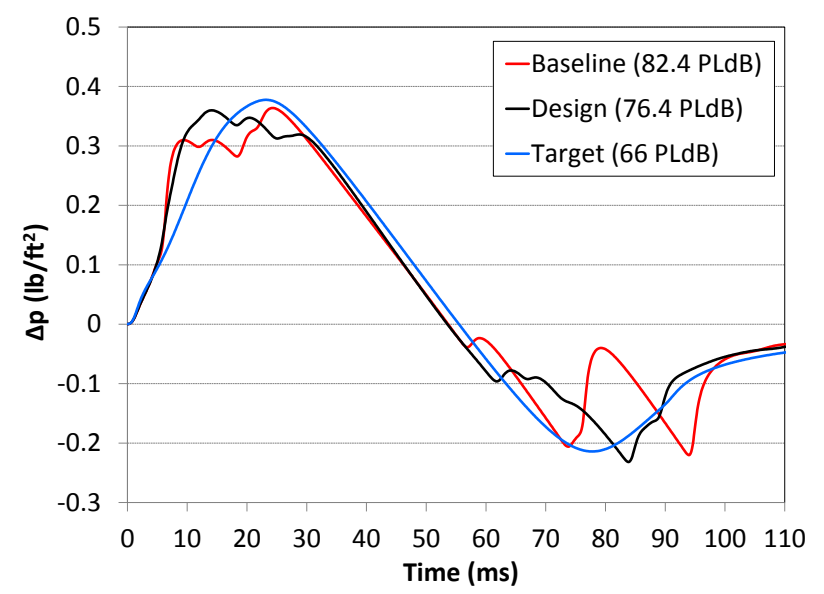

(a) Under-track.

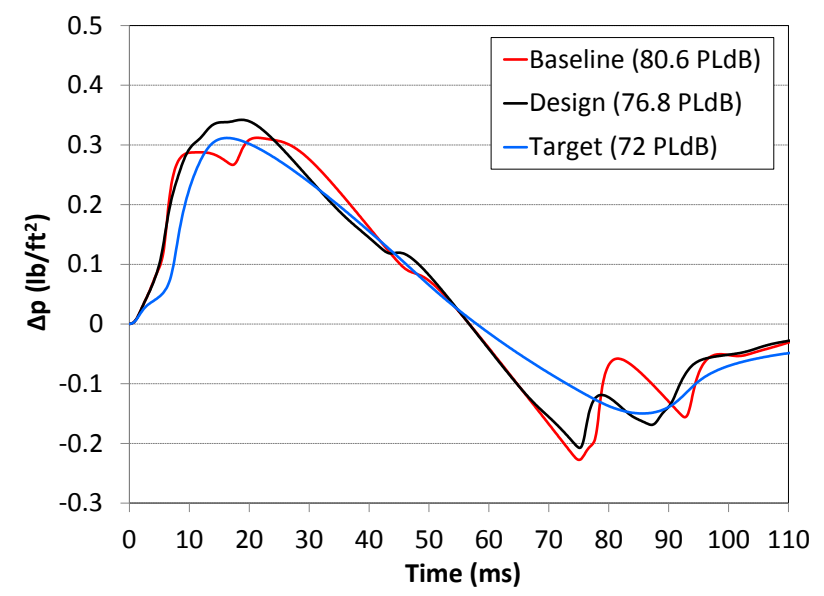

(b) 25 deg off-track.

Figure 9. Comparison of ground $\Delta p$ propagated from $\Delta p / p_{\infty}$ calculated with the design mesh at $h / L=2$ for the baseline and final design configurations.

centerline, and in Fig. 11 for three horizontal tail airfoil sections. The lower height and width (not shown) variables of the three aftmost fuselage sections exhibit significant change from that of the baseline. This occurs because shaping between the end of the wing and the start of the horizontal tail in the $A_{\mathrm{e}}$ domain is limited to volume-based shaping. Shaping based on volume is less effective than with lift when the baseline configuration is relatively far from the target in the $A_{\mathrm{e}}$ domain. The optimization of the horizontal tail shown in Fig. 11 results in a reasonable shape, although, an increase in thickness and camber at the root airfoil is observed, which may result in increased drag. This is not unexpected since aerodynamic efficiency is not considered part of this design effort. Another interesting result of the horizontal tail shaping is that it exhibits a behavior that was also observed during the shaping of the wing. There appears to be a similar reduction in thickness at approximately $20 \%$ chord location of the midspan airfoil section. Despite this observation, the optimization was able to converge before any undesirable feature emerged in the horizontal tail. The contour map of the density adjoint for the final design configuration is shown in Fig. A-2 of the Appendix. This plot shows a significant reduction of the design sensitivity in comparison to that of the baseline configuration shown in Fig. A-1 of the Appendix, and demonstrates the successful convergence toward the low-boom state prescribed by the low-boom targets.

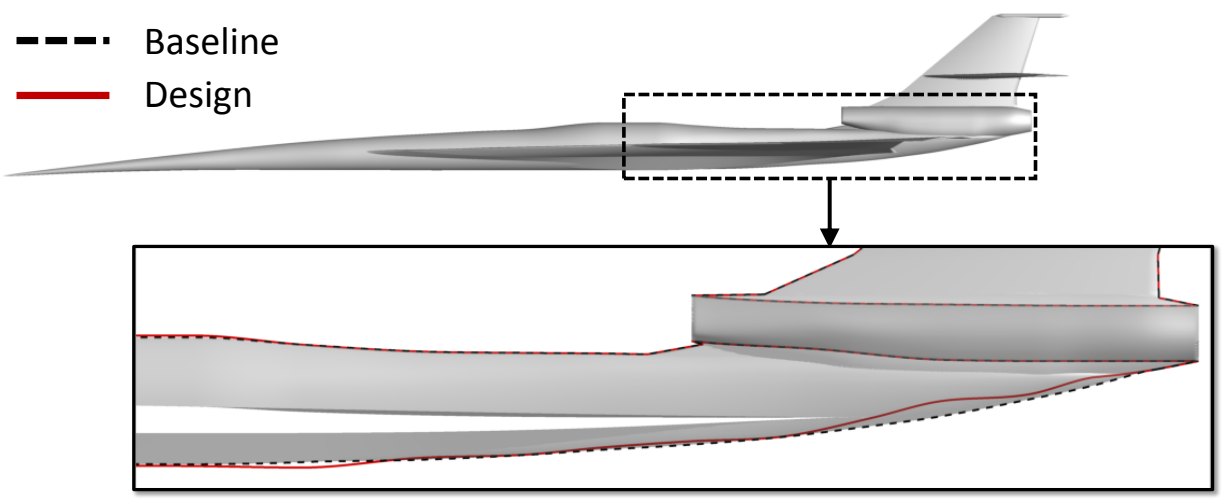

Figure 10. Comparison of fuselage sections for the baseline and final design configurations.

As mentioned in Section IIIC, the trim characteristics are implicitly constrained through the use of a trim-feasible low-boom target, ${ }^{2}$ which is prescribed in the under-track direction. The trim-feasible low-boom target for this configuration is expected to translate the center of pressure $\left(x_{\mathrm{cp}}\right)$ to approximately $x=82 \mathrm{ft}$. The $x_{\mathrm{cp}}$ for the final design configuration was calculated to be located at $x=81.6 \mathrm{ft}$ and corresponds to a forward shift in $x_{\mathrm{cp}}$ of $1.1 \mathrm{ft}$ relative to the baseline configuration. The coefficient of lift (CL) for the final 
design configuration was calculated to be $2.8 \%$ lower than that of the baseline configuration; a start of cruise weight reduction of $600 \mathrm{lb}$.

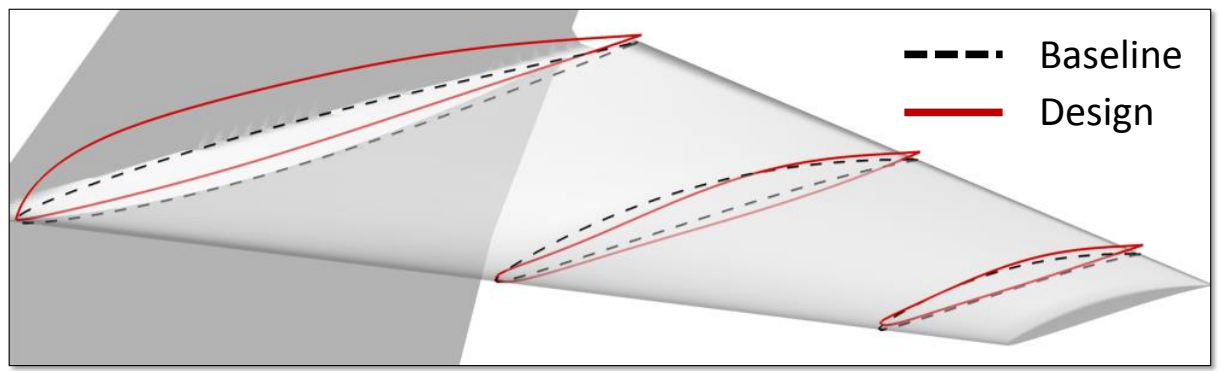

Figure 11. Comparison of horizontal tail airfoil sections for the baseline and final design configurations $(z / x=2$ scaling).

\section{A. Compact Mesh Construction for Reduced Cycle Times}

Off-track target placement was guided by prior under-track experience, in which designs shaped to targets placed at less than $h / L=2$ required significant re-tailoring to be compliant with propagation theory. During the current shaping process, it was observed that off-track signatures could deviate significantly from the specified target while still producing only moderate sonic boom loudness. To first order, loudness for a given off-track signature was dominated by the amplitude of the pressure oscillations about the off-body target, particularly in the aft portion of the signal. While some off-track advantage was expected as a result of increased signal attenuation due to ray path elongation, the degree to which the effect manifested was not.

An alternate target layout is proposed to exploit this phenomenon by bringing the off-track target closer to the body and reducing its weighting within the combined metric. This enables a volume grid with a more compact region of high refinement to be used, in turn reducing the wallclock time required for a function evaluation. A sample mesh is shown in Fig. 12 for the baseline configuration. Mesh stretching and rotation are

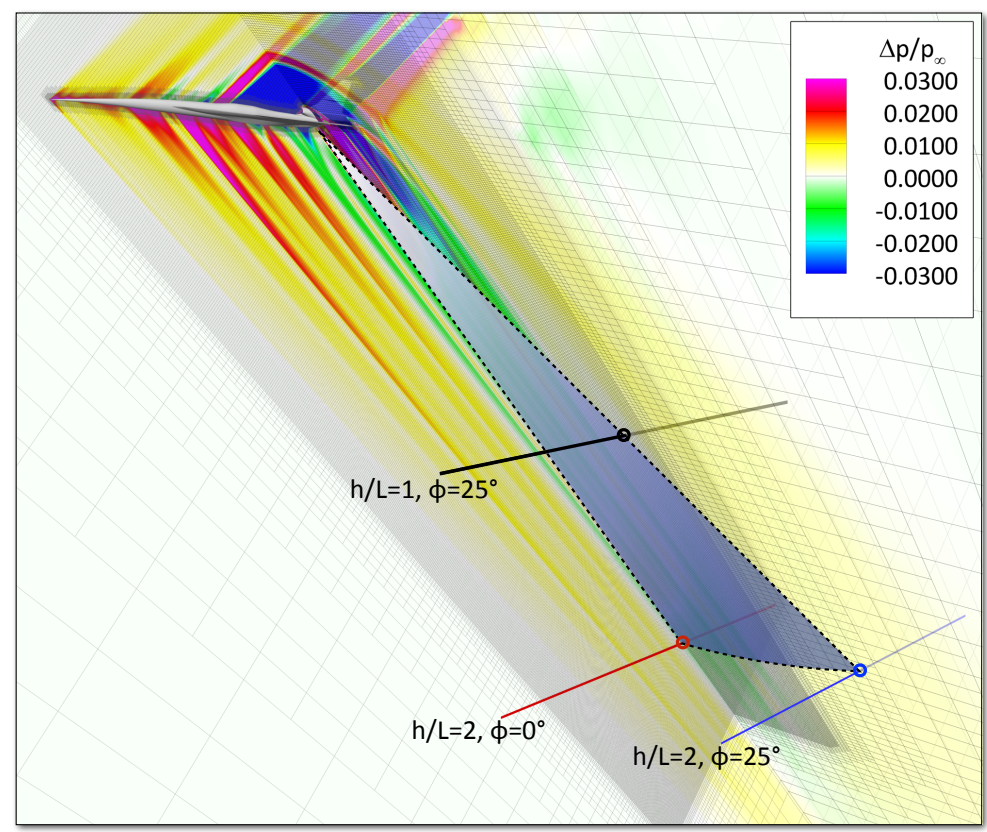

Figure 12. Illustration describing the target positioning for the compact meshing scheme. The off-track, 25 deg azimuth target location is included as reference.

unchanged, as are the nominal cell sizes in the near- and off-body regions. The under-track target is retained at $h / L=2$. The $25 \mathrm{deg}$ off-track target is placed at $h / L=1$, allowing the high-refinement propagation 
region to be halved in size. The prior $h / L=2$ target is included as reference, and is shown to no longer fall within the bounds of the high-refinement region.

A revised design mesh containing 16 million cells is constructed using this compact approach; relative to the previous mesh, function evaluation times are halved. The off-track target is scaled from the previous $h / L=2$ target using the linear $F$-function relation. ${ }^{14}$ An off-track weighting of one-third was found to balance under- and off-track signature loudness as reported on the design mesh. Shape optimization proceeded using the earlier design sequence as a road-map, with shaping parameters activated in similar stages. In addition to increased cycle throughput, the optimizer exhibits improved convergence behavior; this is considered to be a result of reduced discretization error, and increased gradient quality due to the proximity of the off-track target. The baseline and final design $\Delta p / p_{\infty}$ for the under- and off-track signatures are compared against their respective targets in Fig. 13. Fore PLdB has improved from 78.8 to 72 in the under-track direction, and

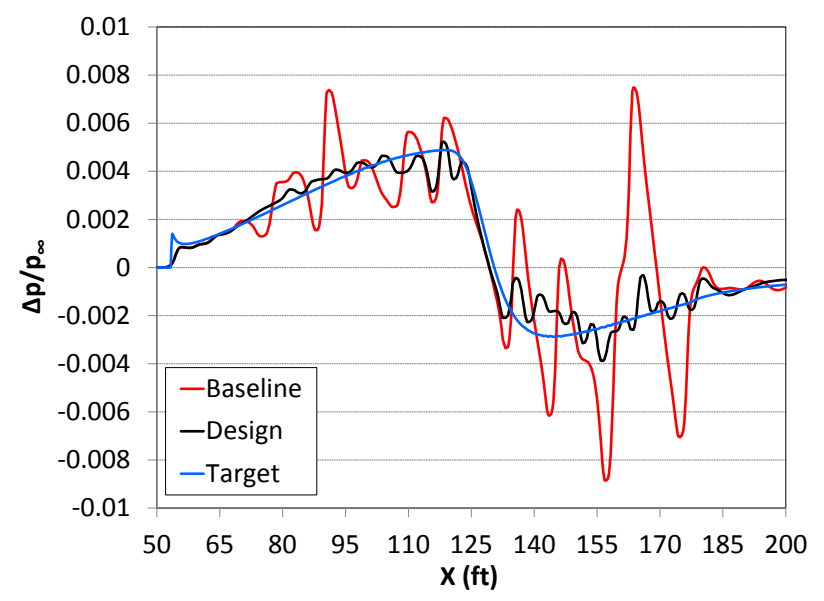

(a) Under-track $(h / L=2)$.

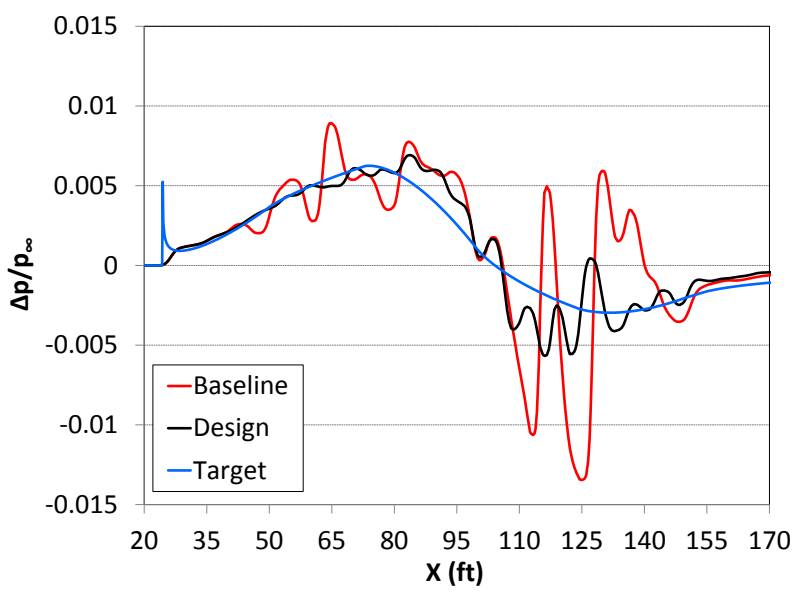

(b) 25 deg off-track $(h / L=1)$.

Figure 13. Comparison of the baseline and final design configurations $\Delta p / p_{\infty}$ developed using the compact mesh approach.

76.5 to 71.5 in the 25 deg off-track. In the aft segment, PLdB has improved from 79.9 to 71 in the undertrack direction, and 75.6 to 73 in the 25 deg off-track. Globally, the under-track signature has decreased from 82.4 PLdB to 73.9 PLdB, while the off-track signature has decreased from 78.8 PLdB to 75.1 PLdB.

Notably, propagation of the off-track signature from $h / L=2$ using the previous design mesh yields fore and aft loudness of 71.5 PLdB and 73.4 PLdB, respectively, along with a global loudness of 75.2 PLdB. The agreement between the loudness predicted at each off-body distance suggests that the initial premise for this compact approach is correct, and reduction in shock amplitudes - rather than precise matching of the target - dominates propagated loudness.

\section{High-Fidelity Verification of Sonic Boom Analysis for Final Design}

The sonic boom properties of the final design are verified across the entire sonic boom carpet using a Cart3D analysis based on an adapted mesh that minimizes discretization error in the computational domain. This verification ensures that the CFD mesh used for design is sufficiently accurate to capture the flowfield, and that the optimizer is not exploiting weaknesses in the analysis.

The high-fidelity sonic boom analysis is conducted at under-track ( $0 \mathrm{deg})$, and 5 additional off-track directions: $7,15,25,35$, and $45 \mathrm{deg}$. In an effort to reduce the numerical dissipation associated with Cartesian meshes for off-track $\Delta p / p_{\infty}$ calculations, the configuration is also rotated so that each azimuthal direction is aligned with the $x-z$ plane at $y=0$. A total of 12 mesh adaptation cycles are conducted to reduce the discretization error at a field sensor located at $h / L=3$, and resulting in a final mesh size of 44 million cells, as shown in Fig. 14 for $\phi=25$ deg.

The off-body $\Delta p / p_{\infty}$ calculated at $h / L=3$ for each azimuth is propagated to the ground with sBOOM, and their corresponding loudness levels are calculated. The PLdB distribution over the sonic boom carpet is shown in Fig. 15. This figure also includes the PLdB values that are calculated from the $\Delta p$ that is 


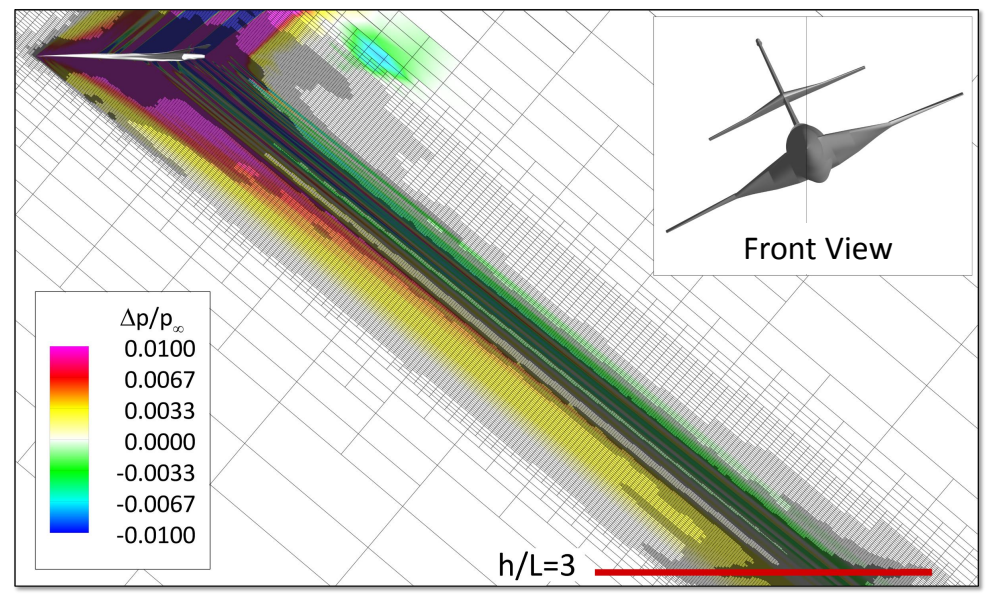

Figure 14. Adapted mesh and Cart3D $\Delta p / p_{\infty}$ solution of the final design configuration at $\phi=25 \mathrm{deg}$.

propagated from the design mesh $\Delta p / p_{\infty}$ at $h / L=2$ for the under-track, and 25 deg off-track directions. This comparison shows that, while the design mesh was sufficiently accurate to capture the sonic boom characteristics of the baseline configuration, it is not able to do the same for the shaped configuration. To better understand the source of this error, the $\Delta p / p_{\infty}$ from the Cart3D solution with an adapted mesh is sampled at $h / L=2$, and propagated to the ground. The PLdB distribution for this case denoted by the dash line in Fig. 15 shows that the propagation from $\Delta p / p_{\infty}$ at $h / L=2$ is not the dominant source of error since it tracks relatively close to the loudness distribution corresponding to the propagation of the $\Delta p / p_{\infty}$ at $h / L=3$.

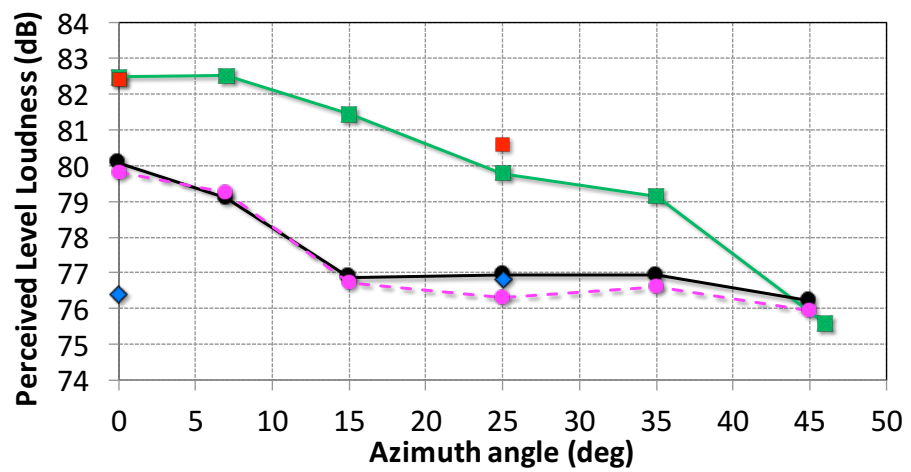

- Baseline (design mesh, propagated from $\mathrm{h} / \mathrm{L}=2$ )

$\diamond$ Design (design mesh, propagated from $\mathrm{h} / \mathrm{L}=2$ )

- -Baseline (adapted mesh, propagated from $h / L=3$ )

$\rightarrow$ Design (adapted mesh, propagated from $\mathrm{h} / \mathrm{L}=3$ )

$\rightarrow$ Design (adapted mesh, propagated from $h / L=2$ )

Figure 15. Off-track loudness distribution of the baseline and final design configurations.

The next likely source for the error is insufficient refinement in the 30 million cell design mesh. The refinement level of the design mesh was increased from 30 million cells to 112 million cells by increasing the number of initial streamwise grid vertices and buffer cell regions, while maintaining the same cell aspect ratio. In addition, the van Leer flux limiter which was used during the design process is replaced with the less dissipative Barth-Jespersen flux limiter.

A comparison of the $\Delta p / p_{\infty}$ calculated at $h / L=2$ for the final design configuration using the 30 million cell design mesh, the 44 million cell adapted mesh, and the 112 million cell mesh is given in Fig. 16. It is worth mentioning that the error objective that is minimized during the Cart3D adjoint-based mesh adaptation places more importance on the stronger shocks than it does on the smaller pressure oscillations. However, the more critical features in $\Delta p / p_{\infty}$, which are not accurately resolved by the design mesh, are the compression 
located at $x=145 \mathrm{ft}$, and the expansion at $x=155 \mathrm{ft}$. Inspection of the more highly refined design mesh consisting of 112 million cells shows that the stronger shock feature, which is observed with the adapted mesh at $x=145 \mathrm{ft}$, is now better resolved. The stronger expansion observed with the adapted mesh at $x=155 \mathrm{ft}$ is also fully resolved. This confirms that the 30 million cell design mesh is too dissipative, and not sufficiently refined to accurately capture the physics of the flowfield for the final design configuration.

Analysis of the $\Delta p / p_{\infty}$ solutions from Fig. 16 in the $A_{\mathrm{e}}$ domain shows that the main variation between the solutions for the design, and adapted meshes appears at an equivalent length $\left(x_{\mathrm{e}}\right)$ of approximately $100 \mathrm{ft}$. The source of the error is traced to the aft region of the final configuration, which is located downstream of the Mach plane corresponding to this $x_{\mathrm{e}}$, as shown in Fig. 17. Three possible solutions for this mesh resolution problem are currently being considered: i) increasing refinement throughout the entire computational domain, ii) addition of a hand-tailored refinement box in the aft region, and iii) performing the design while simultaneously adapting the mesh to reduce discretization error. The latter approach has already been successfully applied to the under-track optimization of the same configuration with powered engines in Ref. 10 .

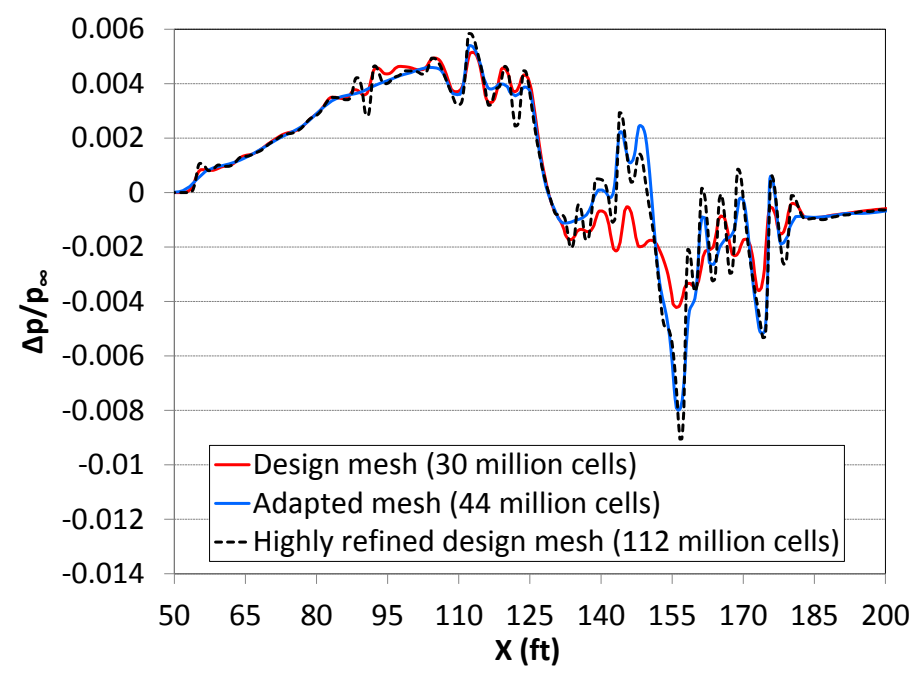

Figure 16. Comparison of under-track $\Delta p / p_{\infty}$ at $h / L=2$ for the final design configuration.

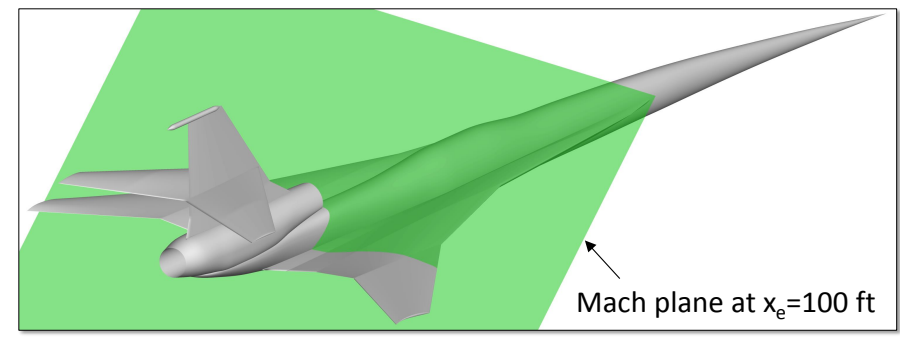

Figure 17. Aft region of the final design configuration where the flowfield is not properly captured.

\section{A. Compact Mesh Design}

The full-carpet verification procedure is repeated for the design developed using the compact mesh approach. Mesh adaptation is configured as for the previous analysis, with the sensor located at $h / L=3$. Predicted loudness across the carpet is plotted in Fig. 18, and compared with the baseline and previous final designs. For consistency, the loudness distribution given in Fig. 18 for the design developed with the compact mesh approach is evaluated with the previous 30 million cell design mesh, and not the compact mesh. Due to the increasing loudness observed beyond $\phi=25 \mathrm{deg}$, an additional case is included to extend the plot to the lateral cutoff angle of $\phi=50 \mathrm{deg}$; this shows a rapid fall-off past $\phi=45 \mathrm{deg}$. Loudness values reported on 


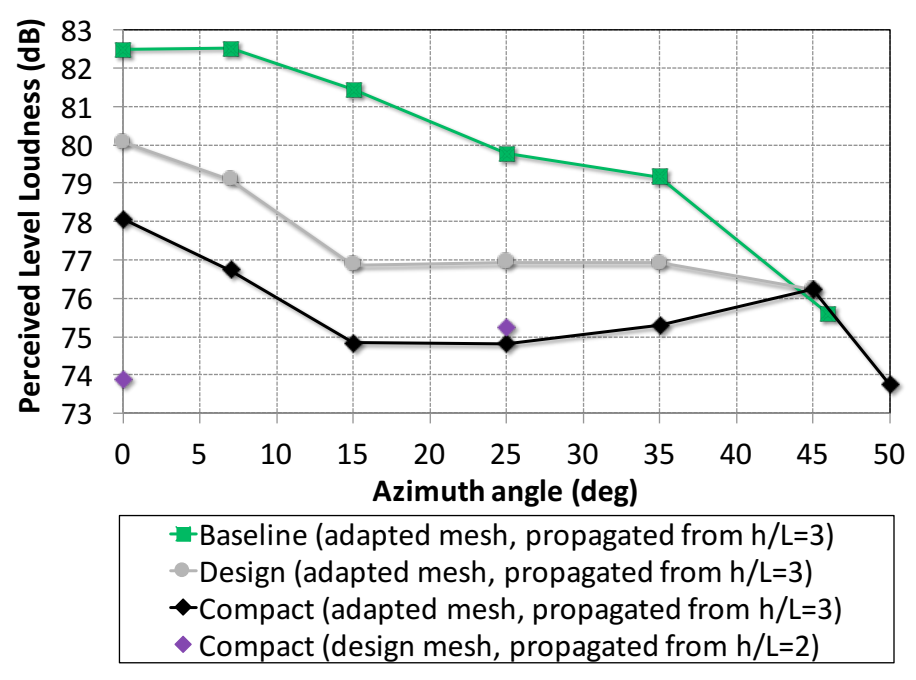

Figure 18. Off-track loudness distribution of the baseline and final design configurations developed using the compact mesh approach.

the compact design mesh are included, and the behavior mirrors the previous case: when evaluated with a high-resolution mesh, under-track loudness increases, and off-track loudness remains essentially unchanged.

The final compact mesh design benefited from experience gained when performing shape optimization with the larger design mesh, and this is credited with the degree to which the full-carpet loudness improves with the compact mesh design. That said, the compelling finding is the speed with which the proposed approach successfully recovered, and subsequently improved upon, the previous result.

\section{Summary}

An approach for low-boom design across the entire sonic boom carpet generated by a demonstrator concept has been presented. Inverse design was used to drive the sonic boom characteristics of the configuration to two equally weighted low-boom targets at the under- and $25 \mathrm{deg}$ off-track directions. This was achieved through use of the Cart3D design framework, which provided gradient information for the coupled optimization problem at the two azimuthal directions.

The hyperbolic form of the governing equations for supersonic flow was leveraged to decompose the optimization problem into smaller subproblems. The decomposition resulted in three shape variable groups, which reduce the complexity of the optimization problem. The first group consisted of variables associated with circular and elliptical sections for the fuselage region aft of the nose cone, and fore of the fuselage-wing intersection. The second group consisted of variables associated with wing airfoil sections, as well as mid and aft fuselage sections. The third group consisted of variables associated with the nacelle, horizontal tail, tail pod, and three aftmost sections of the fuselage, which are located aft of the wing root trailing edge. During the optimization of the wing, it was discovered that, in addition to the constraints imposed on the minimum thickness, area, and leading and trailing edge of the wing, a minimum thickness constraint was needed at the $22 \%$ chord location of the midspan airfoil section.

The design procedures with these three variable groups were shown to be successful in shaping the configuration to match the low-boom targets, and resulted in a reduction of the PLdB distribution across the entire sonic boom carpet. At the 25 deg azimuth angle, which was shown to be the most critical off-track direction during the low-boom optimization conducted strictly below the flight path, ${ }^{10}$ a reduction of approximately 5 PLdB was achieved. The higher fidelity analysis of the sonic boom carpet with adjoint-based mesh adaptation also highlighted a problem in the under-track region with the lower refinement mesh that is used for design. Although the design mesh was shown to accurately capture the sonic boom characteristics of the baseline configuration, it was also shown to be inadequate for the analysis of the final design configuration. In essence, the numerical optimizer was exploiting a weakness in the CFD analysis. The expected improvement of $8.5 \mathrm{PLdB}$, which was calculated from the CFD analysis using the design mesh produced a more modest improvement of $4.5 \mathrm{PLdB}$ with the higher fidelity adapted mesh. 
The problem associated with the design mesh in the under-track region was studied, and demonstrated to be a result of insufficient refinement. This is not unexpected since the mesh was manipulated aggressively to reduce the computational resources needed during design; a product of the significantly large computational domain needed for off-track CFD analysis. The main source of analysis error was traced back in the $A_{\mathrm{e}}$ domain to the aft region of the configuration which includes the nacelle, tail empennage, and aft portion of the wing and fuselage. Three possible solutions were discussed, all of which will inevitably result in increased computational cost.

An alternative approach for the construction of the CFD mesh used for design, and the off-track target layout was presented. This approach was demonstrated to be successful for shaping of the demonstrator concept, while reducing function evaluation times by half. Optimizer convergence was also improved as a result of reduced discretization error, and increased gradient quality due to the proximity of the off-track target. Future work will assess a practical approach to leverage the alternative compact mesh construction with one of the three mesh solutions proposed earlier to improve the CFD analysis fidelity in the under-track region, and the design process will be repeated.

\section{Appendix: Additional Figures}

The combined density adjoint contours for the baseline configuration (Fig. A-1) are provided to show the regions of high sensitivity at this initial state, and for the final design configuration (Fig. A-2) to demonstrate convergence toward the low-boom state prescribed by the low-boom targets.

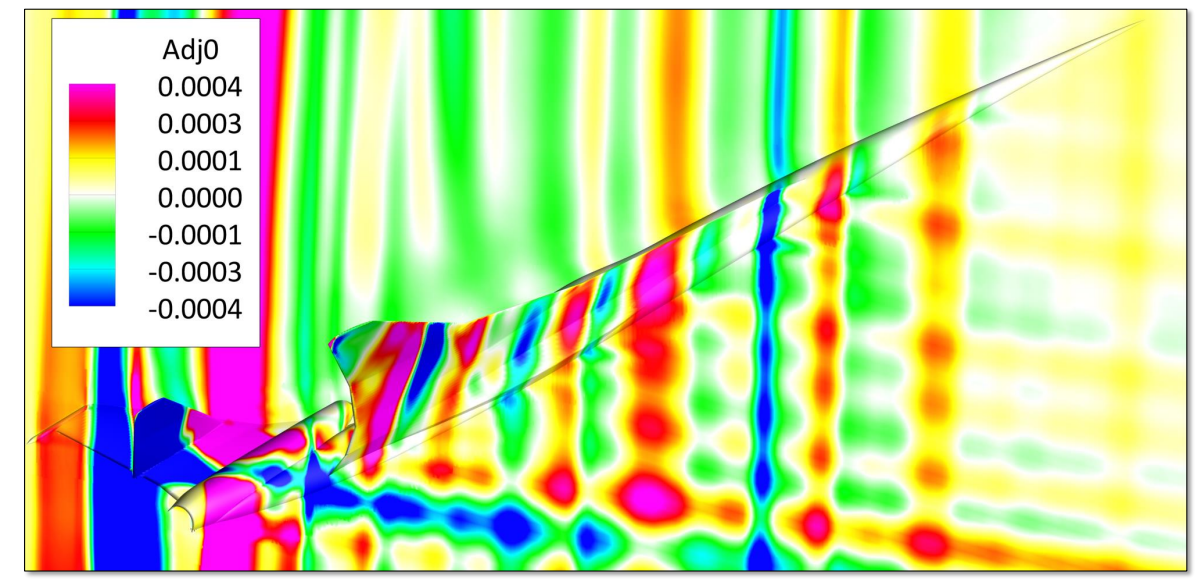

Figure A-1. Combined density adjoint contours of under- and off-track objectives for the baseline configuration.

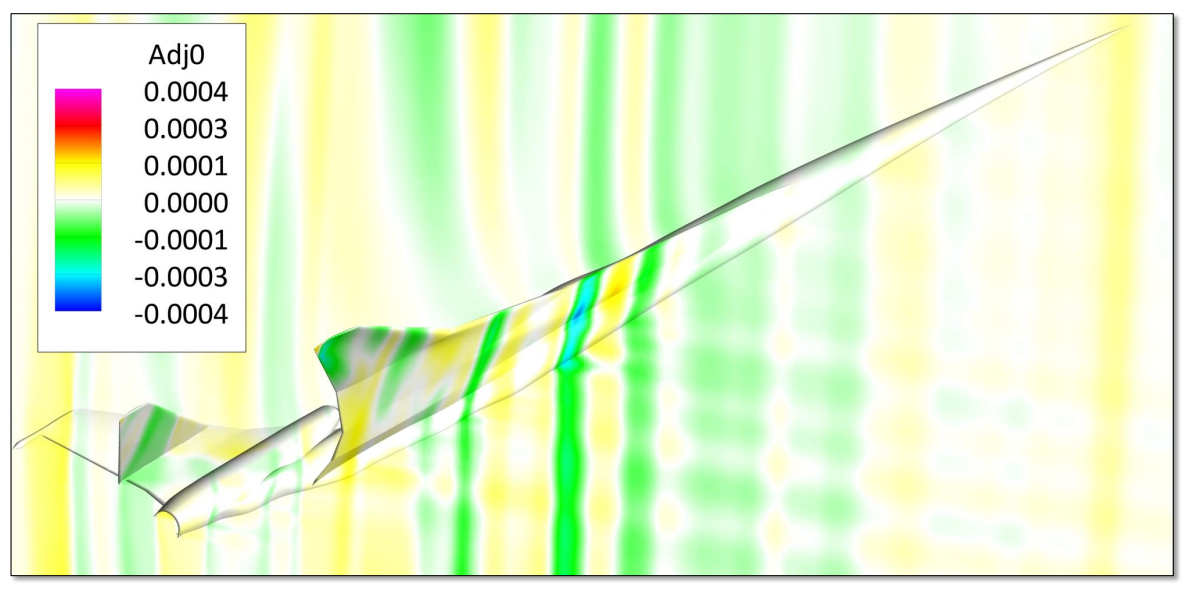

Figure A-2. Combined density adjoint contours of under- and off-track objectives for the final design configuration. 


\section{Acknowledgment}

This work was supported by the NASA Fundamental Aeronautics Program Commercial Supersonic Technology Project through TEAMS2 contract NNL12AA09C, and NIA contract NNL13AA08B.

\section{References}

${ }^{1}$ Trautvetter, C., "NASA Pitches in to End Overland Supersonic Flight Ban," http://www .ainonline.com/aviation-news/ ainalerts/2014-06-17/nasa-pitches-end-overland-supersonic-flight-ban, Aviation International News, posted June 17, 2014.

${ }^{2}$ Ordaz, I., Geiselhart, K. A., and Fenbert, J. W., "Conceptual Design of Low-Boom Aircraft with Flight Trim Requirement," AIAA Paper 2014-2141, June 2014.

${ }^{3}$ Aftosmis, M. J., "Cart3D Resource Website," http://people.nas.nasa.gov/ aftosmis/cart3d, [cited April 2015].

${ }^{4}$ Aftosmis, M. J., Berger, M. J., and Adomavicius, G., "A Parallel Multilevel Method for Adaptively Refined Cartesian Grids with Embedded Boundaries," AIAA Paper 2000-0808, January 2000.

${ }^{5}$ Rallabhandi, S. K., "Advanced Sonic Boom Prediction Using Augmented Burger's Equation," AIAA Paper 2011-1278, January 2011.

${ }^{6}$ Rallabhandi, S. K., "Application of Adjoint Methodology in Various Aspects of Sonic Boom Design," AIAA Paper 20142271, June 2014.

${ }^{7}$ Nemec, M. and Aftosmis, M. J., "Parallel Adjoint Framework for Aerodynamic Shape Optimization of Component-Based Geometry," AIAA Paper 2011-1249, January 2011.

${ }^{8}$ Aftosmis, M. J., Nemec, M., and Cliff, S. E., "Adjoint-Based Low-boom Design with Cart3D," AIAA Paper 2011-3500, June 2011.

${ }^{9}$ Wintzer, M., "Optimization and Adjoint-Based CFD for the Conceptual Design of Low Sonic Boom Aircraft," Ph.d. dissertation, Stanford University, August 2012.

${ }^{10}$ Wintzer, M., Ordaz, I., and Fenbert, J. W., "Under-Track CFD-Based Shape Optimization for a Low-Boom Demonstrator Concept," In preparation, June 2015.

${ }^{11}$ Gill, P. E., Murray, W., and Saunders, M. A., "SNOPT: An SQP Algorithm for Large-Scale Constrained Optimization," SIAM Review, Vol. 47, No. 1, 2005, pp. 99-131.

${ }^{12}$ Kulfan, B. M., "Universal Parametric Geometry Representation Method," Journal of Aircraft, Vol. 45, No. 1, 2008, pp. $142-158$.

${ }^{13}$ Sullivan, B. M., Klos, J., Bnehrle, R. D., McCurdy, D. A., and Haering Jr., E. A., "Human Response to Low-Intensity Sonic Booms Heard Indoors and Outdoors," NASA TM-2010-216685, April 2010.

${ }^{14}$ Whitham, G., "The flow pattern of a supersonic projectile," Communications on pure and applied mathematics, Vol. 5, 1952, pp. 301-348. 